OPEN ACCESS

Edited by:

Lin Lin,

Jiangsu University, China

Reviewed by:

Pei Xu,

South China University of Technology,

China

Yefu Chen

Tianjin University of Science

and Technology, China

*Correspondence:

Youqiang $X u$

xuyouqiang@btbu.edu.cn

Xiuting $L$

lixt@btbu.edu.cn

tThese authors have contributed equally to this work

Specialty section:

This article was submitted to

Food Microbiology,

a section of the journal

Frontiers in Microbiology

Received: 23 November 2021 Accepted: 21 December 2021

Published: 21 February 2022

Citation:

Xu Y, Huang $H, L U H, W u M$,

Lin $M$, Zhang C, Zhao Z, Li W, Zhang C, Li X and Sun B (2022)

Characterization of an Aspergillus niger for Efficient Fatty Acid Ethy Ester Synthesis in Aqueous Phase and the Molecular Mechanism.

Front. Microbiol. 12:820380. doi: 10.3389/fmicb.2021.820380

\section{Characterization of an Aspergillus niger for Efficient Fatty Acid Ethyl Ester Synthesis in Aqueous Phase and the Molecular Mechanism}

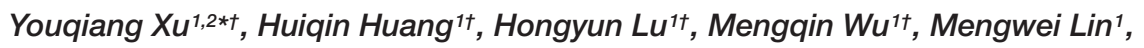
Chunsheng Zhang ${ }^{3}$, Zhigang Zhao ${ }^{3}$, Weiwei Li ${ }^{1}$, Chengnan Zhang ${ }^{1}$, Xiuting $\mathrm{Li}^{1,2 *}$ and Baoguo Sun ${ }^{1,2,4}$

1 Beijing Advanced Innovation Center for Food Nutrition and Human Health, Beijing Technology and Business University, Beijing, China, ${ }^{2}$ Beijing Engineering and Technology Research Center of Food Additives, Beijing Technology and Business University, Beijing, China, ${ }^{3}$ Chengde Qianlongzui Distillery Company, Hebei, China, ${ }^{4}$ Key Laboratory of Brewing Molecular Engineering of China Light Industry, Beijing Technology and Business University, Beijing, China

Fatty acid ethyl esters are important flavor chemicals in strong-flavor baijiu. Microorganisms are the main contributors to ester synthesis during baijiu manufacture. However, the ester synthesis was unstable between batches. This was owing to a limited knowledge of the mechanisms for ester synthesis by microorganisms. In this work, a fatty acid ethyl ester synthesizing Aspergillus niger strain CGMCC (China General Microbiological Culture Collection) 3.4309 was identified. The conversion ratios of ethyl valerate, ethyl caproate, ethyl caprylate, and ethyl caprate were 7.87, 29.20, 94.80, and $85.20 \%$, respectively, under the optimized conditions. A comparison of transcriptomes under the initial and optimized ester synthetic conditions indicated that 23 genes were upregulated in transcription level and encoded enzymes with potential abilities for ester synthesis. Eleven of the enzymes were expressed, and three of them, numbered An605, An1097, and An3131, showed the ability to catalyze fatty acid ethyl ester synthesis under aqueous phase, with capric acid as the preferred substrate. The possible enzymatic catalytic mechanism was proposed based on homology modeling and molecular docking. This study reported for the first time that $A$. niger showed the ability to efficiently catalyze the synthesis of short- and medium-chain fatty acid ethyl esters in aqueous phase, identified the key enzymes, and analyzed the basic enzymatic properties. This is helpful to promote the application of related microorganisms and enzyme resources in the baijiu industry.

Keywords: strong-flavor baijiu, fatty acid ethyl ester, Aspergillus niger, enzyme, aqueous phase, molecular mechanism

\section{INTRODUCTION}

Chinese traditional fermented baijiu is an important composition of Chinese food (Xu et al., 2022b). Strong-flavor baijiu accounts for more than $70 \%$ of the baijiu market share and is a representative of traditional fermented alcoholic beverages (Xu et al., 2021b). Although with a history of thousands of years, strong-flavor baijiu faces problems in the process of modernization. The manufacturing 
processes are still based on an empirical mode, with low efficiency and poor stability between batches (Xu et al., 2022b). The ultimate reason is that the core functional microorganisms and the formation mechanisms of the flavor components are still not yet clear, although some studies reported about baijiu fermentation (Hong et al., 2021; Wu et al., 2021; Xu et al., 2021b). Therefore, it is urgent to clarify the mechanism of synthesizing flavor substances in traditional strong-flavor baijiu, so as to ensure the quality of products. This is an inevitable requirement for the modernization of the baijiu industry (Ye et al., 2021).

Esters are important flavor compounds in baijiu ( $\mathrm{Xu}$ et al., 2022b). The national standard stipulates that strong-flavor baijiu possesses a mixed flavor with the flavor of ethyl caproate as the principal aroma components, indicating that ethyl esters, especially ethyl caproate, are important contributors to the flavor of the product (Xu et al., 2017). Ethyl caproate endows the product with a cellar fragrance and is recognized as a characteristic of high-quality strong-flavor baijiu (Wei et al,, 2020). Other esters such as ethyl acetate, ethyl lactate, and ethyl butyrate are also important esters with fruity and sweet flavors (Xu et al., 2022b). Studies show that microbial metabolism is important for synthesizing fatty acid ethyl esters, including four known microbial synthetic pathways (Kruis et al., 2019). The first route is the synthesis of ethyl acetate catalyzed by alcohol acyltransferases using alcohol and acyl-CoA as the substrates (Kruis et al., 2017). The second one is the synthesis of esters catalyzed by Baeyer-Villiger monooxygenases using 2-ketone as the substrate coupled with the consumption of oxygen and NAD(P)H (Tang et al., 2013). The third one converts alcohol and aldehyde to the corresponding ethyl ester coupled with the co-factor $\mathrm{NAD}(\mathrm{P})^{+}$through hemiacetal dehydrogenation (Yurimoto et al., 2005). The fourth one catalyzes the esterification reaction by the esterifying enzymes such as lipase and esterase using alcohols and acids as the substrates (Xu et al., 2021b). Baijiu flavor analysis indicates that only a slight amount of aldehyde and ketone is produced during the anaerobic fermentation process; thus, the second and third routes do not play a major role for the esterification of ethyl esters (Xu et al., 2022b). The first route is critical to the synthesis of ethyl acetate (Kruis et al., 2017). However, the ratios between ethyl esters have a great influence on the baijiu flavor (Xu et al., 2022b). For strong-flavor baijiu, the concentration of ethyl caproate is significantly higher than that of ethyl acetate ( $\mathrm{Xu}$ et al., 2017). If ethyl caproate is mainly synthesized via alcohol and the acyl-CoA pathway, a large amount of ethyl acetate would be generated as the byproduct. It will lead to the improper ratio of ethyl caproate to ethyl acetate and affects the flavorstyle of strong-flavor baijiu. Therefore, the preferred pathway to synthesize fatty acid ethyl esters of strong-flavor baijiu, especially ethyl caproate, is through the esterification reaction of fatty acids and ethanol. This has also been verified by the fact that the strength of caproic acid-producing strains during fermentation is helpful for the stable synthesis of ethyl caproate (Zou et al., 2018). The main metabolite of caproic acidproducing strains is medium-chain fatty acids dominated by caproic acid and provides a sufficient substrate for esterification (Zou et al., 2018).
Although microorganisms are recognized to play an important role in fatty acid ethyl ester synthesis during baijiu fermentation, studies about the related microorganisms are still scarce. Taking strong-flavor baijiu as an example, many microorganisms are involved in the fermentation process, while only Burkholderia pyrrocinia, Clavispora lusitaniae, Lichtheimia ramosa, and Monascus purpureus were reported with the ability to synthesize fatty acid ethyl esters (Chung et al., 2017; Fan et al., 2020, 2021; Xu et al., 2021b). In addition, although some of these microorganisms promoted ester synthesis in practical application, bottleneck problems existed such as unstable ester concentrations among batches and unclear technical conditions for the actual application of these species (Xu et al., 2022b). The reason is that little is known about the characteristics of the key enzymes that catalyze fatty acid ethyl ester synthesis from microorganisms. Strong-flavor baijiu is mainly produced by solid-state fermentation. Grains [mainly sorghum (Sorghum bicolor $\mathrm{L}$.)] are used as raw materials, and the water content of fermented grains is between 53 and $58 \%$ and can be recognized as an aqueous-phase system (Xu et al., 2017). The best esterification condition of the known enzymes is the organic-phase system (Bollinger et al., 2020). In the aqueous-phase system, these enzymes mainly show the properties for hydrolyzing esters and serve as one of the reasons for the unstable fatty acid ethyl ester production (Ferrer et al., 2015; Bollinger et al., 2020).

In this work, Aspergillus niger from baijiu showed prominent ability for ester synthesis in aqueous phase. For example, under the optimized culture conditions, the conversion ratio of ethyl caprylate was $94.80 \%$ in aqueous phase by A. niger CGMCC (China General Microbiological Culture Collection) 3.4309. Therefore, it was reasonable to conclude that the A. niger CGMCC 3.4309 carried the enzyme for catalyzing fatty acid ethyl ester synthesis in an aqueous-phase system. However, studies on ester synthesis by $A$. niger species were focused on the organic-phase system so far and was obviously different from the aqueous-phase system (Bourne et al., 2004; Mhetras et al., 2010; Cong et al., 2019). The enzyme responsible for catalyzing ester synthesis in the aqueous-phase system was rarely reported. Therefore, the enzymes catalyzing ester synthesis were identified and investigated from A. niger CGMCC 3.4309 in the aqueous-phase system, and the possible catalytic mechanism was proposed. This will help to reveal the molecular mechanism of $A$. niger for fatty acid ethyl ester synthesis in the aqueousphase system and promotes the application of the strain for baijiu fermentation.

\section{MATERIALS AND METHODS}

\section{Materials and Media}

Acetic acid was purchased from Beijing Mairuida Technology Co., Ltd. Propionic acid was purchased from Alfa aesar (China) Chemical Co., Ltd. Butyric acid and caproic acid were purchased from Shanghai Aladdin Biochemical Technology Co., Ltd. Valeric acid was purchased from Beijing J\&K Co., Ltd. Caprylic acid was purchased from Shanghai McLean Biochemical Technology Co., Ltd. Capric acid was purchased from Damas Beta Company. 
Soluble starch was purchased from Xilong Science Co., Ltd. Sucrose, maltose, and glucose were purchased from Fuchen (Tianjin) Chemical Reagent Co., Ltd. Sorghum powder was purchased from Tianjin Lifalong Chemical Technology Co., Ltd. Soybean peptone, tryptone, yeast extract, lactose, and beef extract were purchased from Beijing Aoboxing Biotechnology Co., Ltd. Corn pulp dry powder was purchased from Yuanye Biotechnology Co., Ltd. The potato dextrose agar (PDA) medium contained glucose $(20 \mathrm{~g} / \mathrm{L})$, potato $(200 \mathrm{~g} / \mathrm{L})$, and agar powder $(20 \mathrm{~g} / \mathrm{L})$ with natural $\mathrm{pH}$ value. Fermentation medium contained glucose (100 g/L), soybean powder (10 g/L), ammonium chloride $(12 \mathrm{~g} / \mathrm{L})$, sodium dihydrogen phosphate $(1 \mathrm{~g} / \mathrm{L})$, and magnesium sulfate $(0.2 \mathrm{~g} / \mathrm{L})$ with $\mathrm{pH} 4.5$. All other chemicals were analyticalgrade reagents and commercially available.

\section{Strain Selection}

A total of 10 strains of $A$. niger deposited in CGMCC were investigated for ester synthesis in aqueous phase after being cultured by a fermentation medium for 7 days. Single-factor optimization was carried out to investigate the various factors affecting the production of enzymes for esterification. The strain was cultured on the PDA plate and then transferred to the fermentation medium. It was cultured in a $250 \mathrm{ml}$ flask containing $100 \mathrm{ml}$ fermentation medium at $28^{\circ} \mathrm{C}$ and $200 \mathrm{r} / \mathrm{min}$ for 7 days. The cell culture $(30 \mathrm{ml})$ was transferred into a $50 \mathrm{ml}$ tube for ultrasonic cell crushing. The crushed cell solution was centrifuged at $4^{\circ} \mathrm{C}$ and $9,000 \times g$ for $5 \mathrm{~min}$.

The supernatant was used as the crude enzyme solution to determine the ester synthetic ability. The reaction mixture contained crude enzyme solution $(2 \mathrm{ml}$ ), mixed fatty acids (acetic acid, propionic acid, butyric acid, valeric acid, caproic acid, caprylic acid, and capric acid, $10 \mathrm{mM}$ for each substrate), ethanol (1 M), and a citric acid buffer $(50 \mathrm{mM}, \mathrm{pH} 4.0)$ to the final volume of $10 \mathrm{ml}$. The reaction mixture was kept in a water bath at $30^{\circ} \mathrm{C}$ and $200 \mathrm{r} / \mathrm{min}$ for $12 \mathrm{~h}$. Thereafter, $n$-hexane $(3 \mathrm{ml})$ was added to the reaction system and vortexed for $30 \mathrm{~s}$. The mixture was centrifuged for $10 \mathrm{~min}$ at $4^{\circ} \mathrm{C}$ and $13,000 \times g$, and the upperlayer $n$-hexane was filtered using a $0.22 \mu \mathrm{m}$ membrane and used for gas chromatography (GC) analysis.

\section{Optimization of Fermentation Conditions Optimization of Nitrogen and Carbon Sources}

The six carbon sources were glucose, sucrose, soluble starch, maltose, lactose, and sorghum powder. The seven nitrogen sources were corn slurry dry powder, soybean peptone, yeast extract, beef extract, $\mathrm{NH}_{4} \mathrm{NO}_{3},\left(\mathrm{NH}_{4}\right)_{2} \mathrm{SO}_{4}$, and $\mathrm{NH}_{4} \mathrm{Cl}$. A. niger was cultured using the fermentation medium containing the respective carbon or nitrogen sources in flasks, and kept at $28^{\circ} \mathrm{C}$ and $200 \mathrm{r} / \mathrm{min}$ for 7 days, and used for analysis. The conversion ratios of fatty acids were calculated, and the optimal carbon and nitrogen sources were selected according to the conversion ratios of the substrates.

\section{Optimization of Culture Temperature and Rotation Rates}

After inoculation, the cell cultures were kept at $200 \mathrm{r} / \mathrm{min}$ under different temperatures $\left(20,25,28,30,37\right.$, and $\left.42^{\circ} \mathrm{C}\right)$ for 7 days.
The culture medium inoculated with $A$. niger was kept at $28^{\circ} \mathrm{C}$ for 7 days at different rotation rates $(50,100,150,200,250$, and $300 \mathrm{r} / \mathrm{min}$, respectively). The optimal culture temperature and rotation rates were determined according to the conversion ratios of the substrates.

\section{Transcriptome Analysis}

After the medium composition and culture condition optimization, A. niger CGMCC 3.4309 was cultured under optimized and initial conditions, respectively. Total RNA samples were extracted, respectively, for transcriptome sequencing. Samples were prepared according to the standard protocol by the BGI Co. (Beijing, China). The quality of the sequenced reads was controlled after filtering out the reads with low quality, joint pollution, and unknown bases. Trinity was used to the de novo assembly of clean reads, and then, Tgicl was used to cluster the assembled transcriptome data to remove redundancy and generated unigenes (Supplementary Table 1; Pertea et al., 2003). Thereafter, functional annotation was performed of unigenes. The unigene transcription levels of each sample were calculated and compared. Genes with differentially transcription levels were analyzed by in-depth cluster analysis and functional enrichment analysis. Seven public databases were used for sequence comparison, including non-redundant protein sequence database (NR), nuclear sequence database (NT), Pfam, clusters of orthogonal groups (COGs) for eukaryotic complete genes, the Swiss prot protein sequence database (Swiss prot) and Kyoto Encyclopedia of Genes and Genomes (KEGG), and Gene Ontology (GO). The gene transcription levels were analyzed by using Poisson distribution. The genes with differentiated transcription levels were identified by a strict algorithm between samples. If the number of reads corresponding to gene numbered A is $x$ in a large gene library, the expression of each gene accounts for only a small part of all genes. The distribution of $x$ follows Poisson distribution (Equation 1). Among them, $\lambda$ is the transcriptional copy number determined of gene A (Audic and Claverie, 1997; Li and Dewey, 2011).

$$
p(\mathrm{x})=\frac{e^{-\lambda} \lambda^{x}}{x^{!}}
$$

The total number of reads uniquely aligned to the genome in sample 1 is $\mathrm{N}_{1}$. The total number of reads uniquely aligned to the genome in sample 2 is $\mathrm{N}_{2}$. If the total reads uniquely matched to gene $\mathrm{A}$ in sample 1 is $x$, and in sample 2 is $y$, then the relative transcription divergence of gene $A$ in the two samples can be calculated by the following equation (Equation 2) (Audic and Claverie, 1997; Li and Dewey, 2011). Differentially transcripted genes were identified as genes with varied transcription levels of more than twofold.

$$
2 \sum_{i=0}^{i=y} p(i / x)
$$

$$
\begin{array}{r}
\text { or } 2 \times\left(1-\sum_{i=0}^{i}=y p(i / x) \text { if } \sum_{i=0}^{i=y} p(i / x)>0.5\right. \\
p\left(\frac{\mathrm{y}}{\mathrm{x}}\right)=\left(\frac{N_{2}}{N_{1}}\right)^{y} \frac{(x+y)^{!}}{x^{!} y^{!}\left(1+\frac{N_{2}}{N_{1}}\right)^{(x+y+1)}}
\end{array}
$$




\section{Expression and Identification of Potential Enzymes for Esterification}

\section{Gene Library Construction}

The linearized vector pET-28a $(+)$ was generated by PCR amplification using the primer pair pET28a.f/pET28a.r (Table 1). Genes were amplified through PCR with complementary DNA (cDNA) as the template from the reverse transcription of the total RNA sample using the primer pairs listed in Table 1. The 15-20 bp homologous recombinant sequence regions to the linearized vector pET-28a $(+)$ were added to the $5^{\prime}$ - and $3^{\prime}$-regions of the genes, respectively. Plasmids pET-An408, pET-An605, pETAn923, pET-An1097, pET-An1486, pET-An2017, pET-An2076, pET-An2794, pET-An3040-3, pET-An3131, pET-An3196, pET-An3559, pET-An3936, pET-An5004, and pET-An5100 were constructed according to the protocol of the Vazyme ClonExpress II One Step Cloning Kit (Vazyme Biotech, Nanjing, China). All the constructed plasmids were transferred into E. coli BL21(DE3) to generate the respective transformants as listed in Table 1.

\section{Inducement of Enzyme Expression}

The transformants were inoculated, respectively, to the Lysogeny Broth (LB) medium (added with kanamycin to a final concentration of $40 \mu \mathrm{g} / \mathrm{ml}$ ), and kept at $37^{\circ} \mathrm{C}$ with stirring at $200 \mathrm{r} / \mathrm{min}$ for about $3 \mathrm{~h}$. When the cell density reached the absorbance of $0.6-0.8$ at $\mathrm{OD}_{600 \mathrm{~nm}}$, isopropyl $\beta$-D-1thiogalactopyranoside (IPTG) was added to a final concentration of $0.5 \mu \mathrm{M}$, and kept at $25^{\circ} \mathrm{C}$ with stirring at $200 \mathrm{r} / \mathrm{min}$ for about $20 \mathrm{~h}$. The cell culture was centrifuged at $13,000 \times \mathrm{g}$ for $5 \mathrm{~min}$ at $4^{\circ} \mathrm{C}$, and washed twice with a Tris- $\mathrm{HCl}$ buffer $(50 \mathrm{mM}$, $\mathrm{pH}$ 7.0). Cells were finally suspended by the Tris- $\mathrm{HCl}$ buffer and disrupted with a sonifier (Ningbo Xinzhi, Ningbo, China), and centrifuged thereafter. The supernatant was used as the crude enzyme solution for analysis. The crude enzyme solution was analyzed by sodium dodecyl sulfate - polyacrylamide gel electrophoresis (SDS-PAGE) to verify enzyme expression.

\section{Esterification Using Whole Cell Catalysis}

Whole cell catalysis was used for enzymatic esterification analysis. After the addition of IPTG and being cultured for $20 \mathrm{~h}$, Triton X-100 was added to the cell culture to the final concentration of $0.3 \%(\mathrm{v} / \mathrm{v})$. Ethanol was added to the final concentration of $5.0 \mathrm{~g} / \mathrm{L}$, and valeric acid, caproic acid, caprylic acid, and capric acid were added to the final concentration of $10 \mathrm{mM}$ for each chemical, and kept at $28^{\circ} \mathrm{C}$ and $250 \mathrm{r} / \mathrm{min}$ for $8 \mathrm{~h}$. $n$-Hexane was added to the reaction mixture by $10 \mathrm{ml}$, vortexed for $30 \mathrm{~s}$, and transferred to a $50 \mathrm{ml}$ tube for centrifugation at $4^{\circ} \mathrm{C}$ and $13,000 \times g$ for $10 \mathrm{~min}$. The upper layer $n$-hexane was filtered using a $0.22 \mu \mathrm{m}$ membrane and used for GC analysis.

\section{Enzyme Purification and Property Analysis}

The crude enzyme solution was purified using an Ni Sepharose high performance (HP) column (1 ml; GE, Uppsala, Sweden) with a phosphate buffer $(50 \mathrm{mM}, \mathrm{pH} 7.4,400 \mathrm{mM} \mathrm{NaCl})$ and varied concentrations of imidazole using ÄKTA Fast Protein
Liquid Chromatography purification system (GE, Uppsala, Sweden). The purified enzyme was confirmed by SDS-PAGE and quantified by Coomassie brilliant blue dyeing method using bovine serum albumin as the standard.

The enzyme was used for esterification with ethanol and fatty acids as the substrates. The reaction system contained the known amount of purified enzyme solution, capric acid (a final concentration of $10 \mathrm{mM}$ ), ethanol $(1 \mathrm{M})$, and reaction buffer to a final volume of $1 \mathrm{ml}$. The effect of $\mathrm{pH}$ on enzyme activity was analyzed with $\mathrm{pH}$ values from 3.0 to $8.0\left(\mathrm{Na}_{2} \mathrm{HPO}_{4} /\right.$ citric acid buffer, $50 \mathrm{mM}$ ) at $30^{\circ} \mathrm{C}$. For $\mathrm{pH}$ stability analysis, the enzyme solution was added to an $\mathrm{Na}_{2} \mathrm{HPO}_{4}$ /citric acid buffer $(50 \mathrm{mM}$, pH 4.0 ), and kept for 0.5 or $1.0 \mathrm{~h}$. Thereafter, the reaction mixture was prepared and kept at $30^{\circ} \mathrm{C}$ for $1.0 \mathrm{~h}$. The optimum temperature was analyzed after the reaction mixture was kept at different temperatures in an $\mathrm{Na}_{2} \mathrm{HPO}_{4}$ /citric acid buffer ( $50 \mathrm{mM}, \mathrm{pH} 4.0$ ). For temperature stability analysis, the enzyme solution was kept at $30^{\circ} \mathrm{C}$ for 0.5 or $1.0 \mathrm{~h}$. Thereafter, the reaction mixture was prepared in an $\mathrm{Na}_{2} \mathrm{HPO}_{4}$ /citric acid buffer (50 mM, pH 4.0), and kept for $1.0 \mathrm{~h}$. When detecting the effects of ions or surfactants on enzyme activity, a $2 \mathrm{mM}$ ion or surfactant was added to the reaction mixture containing capric acid $(10 \mathrm{mM})$ and ethanol (1 M), and kept at $30^{\circ} \mathrm{C}$ for $1 \mathrm{~h}$.

\section{Homology Modeling and Molecular Docking}

Homology modeling was performed using Discovery Studio Modeler V2019 software. The appropriate reference enzymes were selected from the PDB_nr95 database according to the sequence similarity. The secondary structure of the enzyme was predicted based on the sequence alignment with the reference enzymes. Thereafter, the three-dimensional (3D) structure model was constructed and the quality of the model was evaluated using Ramachandran plot analysis. The enzyme was set as the receptor and used for molecular docking. All the ligands (caproic acid, caprylic acid, capric acid, ethyl caproate, ethyl caprylate, and ethyl caprate) were downloaded from National Center for Biotechnology Information (NCBI) PubChem Compound.

Reference models were searched by similarities using the sequences of proteins as the templates in the Protein Data Bank (PDB) database. Sequence alignment was analyzed for finding the catalytic active sites. Molecular docking was carried out using Discovery Studio CDOCKER (V2019). The grid option tool was opened under the command of grid box to modify the receptor protein, including hydrogenation and charge balance. The size of the ligand binding pocket of the receptor enzyme was jointly determined and set as the center of the binding site. By calculating the equivalent comprehensive scores of the repulsion, hydrogen bond, hydrophobic interaction, and molecular flexibility of the receptor substrate complex, the affinity of the ligand and receptor was evaluated, and outputted in the format of the binding energy score. The binding energy was the core parameter to measure whether the substrate could effectively bind to the receptor molecule. A higher affinity of the ligand and receptor was usually related to a lower binding energy score (Williams et al., 2004). The proteins with sequence similarity lower than 
TABLE 1 | Strains, vectors, and primers used in this work.

\begin{tabular}{|c|c|c|}
\hline Strain & Characteristic & References \\
\hline Escherichia coli BL21(DE3) & Used as host strain & Invitrogen \\
\hline E. coli BL21/pET-28a(+) & E. coli BL21(DE3) harboring expression vector pET-28a(+) & This work \\
\hline E. coli BL21/pET-An408 & E. coli BL21(DE3) harboring expression vector pET-An408 & This work \\
\hline E. coli BL21/pET-An605 & E. coli BL21(DE3) harboring expression vector pET-An605 & This work \\
\hline E. coli BL21/pET-An923 & E. coli BL21(DE3) harboring expression vector pET-An923 & This work \\
\hline E. coli BL21/pET-An1097 & E. coli BL21(DE3) harboring expression vector pET-An1097 & This work \\
\hline E. coli BL21/pET-An1486 & E. coli BL21(DE3) harboring expression vector pET-An1486 & This work \\
\hline E. coli BL21/pET-An2017 & E. coli BL21(DE3) harboring expression vector pET-An2017 & This work \\
\hline E. coli BL21/pET-An2076 & E. coli BL21(DE3) harboring expression vector pET-An2076 & This work \\
\hline E. coli BL21/pET-An2794 & E. coli BL21(DE3) harboring expression vector pET-An2794 & This work \\
\hline E. coli BL21/pET-An3040-3 & E. coli BL21(DE3) harboring expression vector pET-An3040-3 & This work \\
\hline E. coli BL21/pET-An3131 & E. coli BL21(DE3) harboring expression vector pET-An3131 & This work \\
\hline E. coli BL21/pET-An3196 & E. coli BL21(DE3) harboring expression vector pET-An3196 & This work \\
\hline E. coli BL21/pET-An3559 & E. coli BL21(DE3) harboring expression vector pET-An3559 & This work \\
\hline E. coli BL21/pET-An3936 & E. coli BL21(DE3) harboring expression vector pET-An3936 & This work \\
\hline E. coli BL21/pET-An5004 & E. coli BL21(DE3) harboring expression vector pET-An5004 & This work \\
\hline E. coli BL21/pET-An5100 & E. coli BL21(DE3) harboring expression vector pET-An5100 & This work \\
\hline Vector & Characteristic & References \\
\hline pET-28a $(+)$ & pMB1 replicon, Kanr, carried PT7 promoter & Novagen \\
\hline pET-An408 & pET-28a(+) inserted with the gene numbered An408 & This work \\
\hline pET-An605 & pET-28a(+) inserted with the gene numbered An605 & This work \\
\hline pET-An923 & pET-28a(+) inserted with the gene numbered An923 & This work \\
\hline pET-An1097 & pET-28a(+) inserted with the gene numbered An1097 & This work \\
\hline pET-An1486 & pET-28a(+) inserted with the gene numbered An1486 & This work \\
\hline pET-An2017 & pET-28a(+) inserted with the gene numbered An2017 & This work \\
\hline pET-An2076 & pET-28a(+) inserted with the gene numbered An2076 & This work \\
\hline pET-An2794 & pET-28a(+) inserted with the gene numbered An2794 & This work \\
\hline pET-An3040-3 & pET-28a(+) inserted with the gene numbered An3040-3 & This work \\
\hline pET-An3131 & pET-28a(+) inserted with the gene numbered An3131 & This work \\
\hline pET-An3196 & pET-28a(+) inserted with the gene numbered An3196 & This work \\
\hline pET-An3559 & pET-28a(+) inserted with the gene numbered An3559 & This work \\
\hline pET-An3936 & pET-28a(+) inserted with the gene numbered An3936 & This work \\
\hline pET-An5004 & pET-28a(+) inserted with the gene numbered An5004 & This work \\
\hline pET-An5100 & pET-28a(+) inserted with the gene numbered An5100 & This work \\
\hline Primer name $^{a}$ & Sequence $\left(5^{\prime}-3^{\prime}\right)^{b}$ & \\
\hline pET28a.f & ATCGCACTCGAGCACCACC & \\
\hline pET28a.r & CATGGTATATCTCCTTCTTA & \\
\hline An408.f & TAAGAAGGAGATATACCATGATGCTTGGCCGCTTGGAG & \\
\hline An408.r & GGTGGTGCTCGAGTGCGATATCCCATCGAGTGAAATA & \\
\hline An605.f & TAAGAAGGAGATATACCATGATGCATACTCCATATCTTC & \\
\hline An605.r & GGTGGTGCTCGAGTGCGATGTAGCTCCGATCAATCCAG & \\
\hline An923.f & 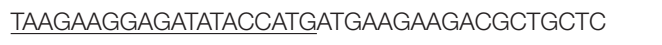 & \\
\hline An923.r & GGTGGTGCTCGAGTGCGATCTCCACGACCACAACGTC & \\
\hline An1097.f & 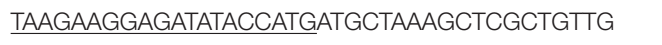 & \\
\hline An1097.r & GGTGGTGCTCGAGTGCGATTCGCCAGTACGCGTTGGCC & \\
\hline An1486.f & TAAGAAGGAGATATACCATGATGCATIITCTCATTCT & \\
\hline An1486.r & GGTGGTGCTCGAGTGCGATCACCCCCAACTCACTCGC & \\
\hline An2017.f & TAAGAAGGAGATATACCATGATGCTAGACGTGTCACTTG & \\
\hline An2017.r & GGTGGTGCTCGAGTGCGATCATCATCCAAACCCCAAC & \\
\hline
\end{tabular}


TABLE 1 | (Continued)

\begin{tabular}{|c|c|}
\hline Primer name ${ }^{a}$ & Sequence $\left(5^{\prime}-3^{\prime}\right)^{b}$ \\
\hline An2076.f & TAAGAAGGAGATATACCATGATGACCGCGTATGGCGGC \\
\hline An2076.r & GGTGGTGCTCGAGTGCGATAATCTCCATTTCCGGCGC \\
\hline An2794.r & GGTGGTGCTCGAGTGCGATGTAAAGAGTACCGCAGTAC \\
\hline An3040-3.f & TAAGAAGGAGATATACCATGATGGATTACTCCGTCAAC \\
\hline An3040-3.r & GGTGGTGCTCGAGTGCGATTGACCGTACATGGTAACT \\
\hline An3196.f & TAAGAAGGAGATATACCATGATGGGGATAAAGCCAAAG \\
\hline An3196.r & GGTGGTGCTCGAGTGCGATAGGCGTCCTAGCACCTTC \\
\hline An3559.f & TAAGAAGGAGATATACCATGATGGCCACTCTTAGAATG \\
\hline An3559.r & GGTGGTGCTCGAGTGCGATTACTCCGGCCTCTGCTCG \\
\hline An3936.f & TAAGAAGGAGATATACCATGATGCAGCTTCAATTCATC \\
\hline An5100.r & 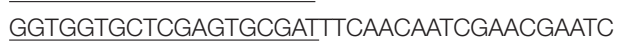 \\
\hline An163-2.f & TAAGAAGGAGATATACCATGATGCGGCTCGCGCCCCAG \\
\hline An163-2.r & 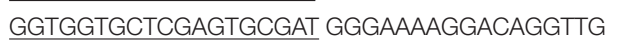 \\
\hline An163-6.f & TAAGAAGGAGATATACCATGATGGCCTACATGCTGTAC \\
\hline An163-6.r & 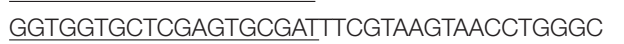 \\
\hline An286.f & TAAGAAGGAGATATACCATGATGATCGCTCCCAAAATC \\
\hline An286.r & 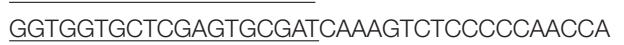 \\
\hline An1556-4.f & TAAGAAGGAGATATACCATGATGAAGCCCGGGTATTGC \\
\hline An1556-4.r & GGTGGTGCTCGAGTGCGATTTGAGGTACCCTCTCCGA \\
\hline An2505.f & TAAGAAGGAGATATACCATGATGAAGGATGATTACAAC \\
\hline An2505.r & GGTGGTGCTCGAGTGCGATATAAGGCACATACTCCTC \\
\hline
\end{tabular}

a.f means sense prime, .r means antisense prime.

bUnderlined region is the homologous recombinant sequence to the ends of $5^{\prime}$ - or $3^{\prime}$-terminal of the linearized pET-28a(+) vector.

$25 \%$ were modeled using the online version of RoseTTAFold ${ }^{1}$ (Baek et al., 2021). Then, the Dali server ${ }^{2}$ (Holm and Laakso, 2016) was used to compare newly generated $3 \mathrm{D}$ models against structures in the PDB.

\section{Analytical Methods}

The concentrations of the fatty acid esters were quantified by Agilent 7890B GC (Agilent, Santa Clara, CA, United States) equipped with a $19091 \mathrm{~N}-213 \mathrm{I}$ column $(30 \mathrm{~m} \times 0.32 \mathrm{~mm} \times 0.50 \mu \mathrm{m}$; Agilent, Santa Clara, CA, United States), a flame ionization detector, and an autosampler. The standard chemicals were serial-diluted and detected in triplicate to generate the standard curve. The GC analysis used an oven temperature program: $40^{\circ} \mathrm{C}$ for $5 \mathrm{~min}$, increased to $170^{\circ} \mathrm{C}$ at $8^{\circ} \mathrm{C} / \mathrm{min}$, maintained for $10 \mathrm{~min}$, increased to $240^{\circ} \mathrm{C}$ at

${ }^{1}$ https://robetta.bakerlab.org/

${ }^{2}$ http://ekhidna2.biocenter.helsinki.fi/dali/oldstyle.html $8^{\circ} \mathrm{C} / \mathrm{min}$, and maintained for $5 \mathrm{~min}$. The carrier gas was nitrogen with a flow rate of $1.0 \mathrm{ml} / \mathrm{min}$. The injection volume was $1.0 \mu \mathrm{l}$ and was delivered in splitless mode. Origin 8.0 software was used for statistical analysis.

\section{RESULTS AND DISCUSSION}

\section{Identification and Optimization of Aspergillus niger Species Synthesizing Fatty Acid Ethyl Esters}

Microorganisms are one of the deciding factors affecting baijiu quality (Jin et al., 2017; Wang et al., 2020, 2021). They not only metabolically synthesize flavor chemicals but also degrade some of the harmful chemicals in the raw materials, thus contributing greatly to the flavor and quality of the products (Xu Y. Q. et al., 2020; Xu et al., 2021a, 2022a). A. niger was generally recognized as an important functional microorganism 
in baijiu manufacture (Wu et al., 2015). Previous studies indicated its performance on the liquefying and saccharifying of the raw materials in the baijiu fermentation process ( $\mathrm{Wu}$ et al., 2015; Jain and Katyal, 2018). Scarcely any studies investigated its function on esterification in an aqueous-phase system. In this work, the species also showed the esterification ability (Figure 1). A. niger species numbered CGMCC 3.489, CGMCC 3.1858, CGMCC 3.2169, CGMCC 3.2103, CGMCC 3.4309, CGMCC 3.2113, CGMCC 3.2130, CGMCC 3.0875, CGMCC 3.2133, and CGMCC 3.0876 were investigated, and the strain numbered CGMCC 3.4309 showed a superior esterification property. The conversion ratios of ethyl valerate, ethyl caproate, ethyl caprylate, and ethyl caprate were $2.23,4.89,44.30$, and $28.20 \%$ (Figure 1), respectively.

Single-factor experiments were used to investigate the fermentation conditions and medium components that might affect the ester synthesis of $A$. niger CGMCC 3.4309, such as the type of carbon and nitrogen sources, fermentation temperature, and rotation rate. Seven nitrogen sources were analyzed including $\mathrm{NH}_{4} \mathrm{Cl}$, soybean peptone, yeast extract, beef extract, $\mathrm{NH}_{4} \mathrm{NO}_{3}$, $\left(\mathrm{NH}_{4}\right)_{2} \mathrm{SO}_{4}$, and corn slurry dry powder (Figure 1B). $\left(\mathrm{NH}_{4}\right)_{2} \mathrm{SO}_{4}$ was the preferred nitrogen source. The conversion ratios of ethyl valerate, ethyl caproate, ethyl caprylate, and ethyl caprate were $2.94,16.90,95.00$, and $62.00 \%$ (Figure 1B), respectively. Six kinds of carbon sources were investigated, including glucose, sucrose, soluble starch, maltose, lactose, and sorghum powder. Lactose served as the preferred carbon source, and the conversion ratios of ethyl valerate, ethyl caproate, ethyl caprylate, and ethyl caprate were $4.74,18.70,96.60$, and $70.40 \%$ (Figure 1C), respectively. The optimal culture temperature was investigated, and the preferred temperature was $28^{\circ} \mathrm{C}$ according to the ester conversion ratio. The respective conversion ratios were $6.90,24.50,89.80$, and $72.00 \%$ (Figure 1D). The rotation rate can affect the enzyme production by affecting the dissolved oxygen concentration of fermentation medium and the dispersion of fungal species (Feng et al., 2003). The preferred rotation rate for producing enzymes responsible for esterification was $250 \mathrm{r} / \mathrm{min}$ after an optimization of rotation rate. The conversion ratios of ethyl valerate, ethyl caproate, ethyl caprylate, and ethyl caprate were 7.87, 29.20, 94.80, and $85.20 \%$ (Figure 1E), respectively.

In this study, A. niger CGMCC 3.4309 showed a good performance on esterification by determining the conversion ratios of ethyl valerate, ethyl caproate, ethyl caprylate, and ethyl caprate. The conversion ratios of the four ethyl esters increased for 3.53, 5.97, 2.14, and 3.02 folds, respectively, after an optimization of cultural conditions (Figure 1F). Meanwhile, the concentrations of the reaction substrates were added according to the concentrations of such chemicals during the actual baijiu fermentation process (Xu et al., 2021b). The concentration of caprylic acid or capric acid each was $10 \mathrm{mM}$, and the conversion ratios already reached 44.30 and $28.20 \%$, respectively, before optimization. With enough substrates, the conversion ratios of ethyl caprylate and ethyl caprate could be further improved under optimized conditions.

This was the first identification of an $A$. niger species for efficient fatty acid ethyl ester synthesis in an aqueous-phase system. So far, only a few studies reported the ester synthesis of fungal species from baijiu in the aqueous phase system, such as M. purpureus and C. lusitaniae (Fan et al., 2021; Xu et al., 2021b). It was not a frequently identified property of the microorganisms from baijiu. This served as the reason for the unstable ester synthesis during baijiu fermentation. Even the strains of the same species showed different performances for esterification such as the M. purpureus species as reported in our previous work (Xu C. et al., 2020). This indicated the genetic divergences between the strains of the same species. The strains with efficient esterification properties might harbor specific enzymes responsible for esterification. A. niger CGMCC 3.4309 could simultaneously synthesize ethyl valerate, ethyl caproate, ethyl caprylate, and ethyl caprate in aqueous phase. Thus, it was necessary to investigate the possible enzymes for esterification.

\section{Screening of the Possible Enzyme for Ester Synthesis in Aqueous-Phase System}

A BGIseq-500 platform was used to assemble the transcriptome data and generated a total of 24,319 unigenes (Supplementary Table 1). The unigenes were annotated to the seven functional databases NR, NT, Swissprot, COG, KEGG, GO, and Pfam, respectively. Finally, 22,396 (92.09\%) unigenes were annotated by the NR database, 23,223 (95.49\%) by NT, 14,469 (59.50\%) by Swissprot, 12,901 (53.05\%) by COG, 15,484 (63.67\%) by KEGG, $16,476(67.75 \%)$ by GO, and $16,153(66.42 \%)$ by Pfam. The genes with transcriptional divergences were analyzed (Supplementary Figure 1). A total of 23 genes were upregulated in transcription and encoded the proteins with the potential for esterification (Table 2 and Supplementary Table 2). Among them, 15 genes were cloned, including An408, An605, An923, An1097, An1486, An2017, An2076, An2794, An30403, An3131, An3196, An3559, An3936, An5004, and An5100 (Supplementary Figure 2a). SDS-PAGE indicated 11 of the enzymes were expressed, except An2017, An3196, An3559, and An5004 (Supplementary Figure 2b).

Generally, enzymatic esterification preferred an organic-phase reaction system (Bollinger et al., 2020). Only some studies investigated an enzymatic synthesis of fatty acid esters in an aqueous-phase reaction system (Sun et al., 2012; Sun and Liu, 2015). In this study, some of the enzymes from A. niger CGMCC 3.4309 could synthesize fatty acid ethyl esters in an aqueousphase reaction system. Esterification reaction showed that the enzyme encoded by An605 could synthesize ethyl caproate and ethyl caprate. The enzyme encoded by An1097 could synthesize ethyl caproate, ethyl caprylate, and ethyl caprate. The enzyme encoded by An3131 could synthesize ethyl caprylate and ethyl caprate (Figure 2). The results indicated that the esterification performance of $A$. niger CGMCC 3.4309 were the co-works of several enzymes (Figure 2).

\section{Enzymatic Property Investigation}

The substrate specificity of ester synthetic enzyme is determined by the activity of catalyzing the synthesis of esters with different chain length fatty acids as the substrates. In this study, An605 and An3131 were purified and the substrate specificity were 

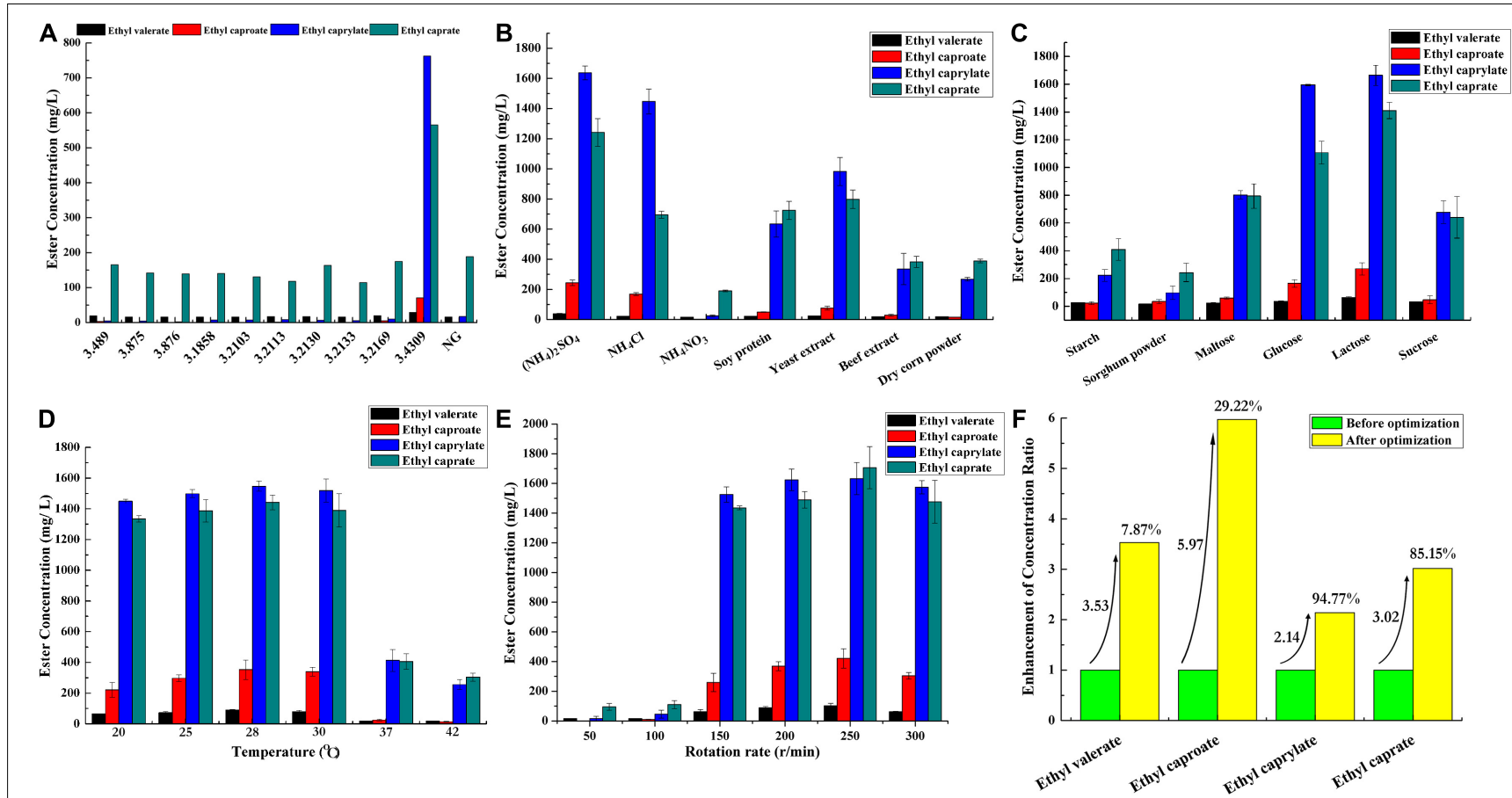

FIGURE 1 | Identification and optimization of cultural conditions of $A$. niger for fatty acid ethyl ester synthesis. (A) Comparison of the esterification properties of different $A$. niger strains, NG, negative control, the medium without inoculation of $A$. niger. (B) Nitrogen source optimization for culturing $A$. niger CGMCC 3.4309 . (C) Carbon source optimization for culturing A. niger CGMCC 3.4309. (D) Temperature optimization for culturing A. niger CGMCC 3.4309. (E) Rotation rate optimization for culturing A. niger CGMCC 3.4309. (F) The conversion rate comparison of the fatty acid ethyl esters by A. niger CGMCC 3.4309 before and after optimization.

investigated, while An1097 was failed to purify (Supplementary Figure 3). The substrate specificities of An605 and An3131 were the same, and both of them showed preference toward long-chain fatty acid capric acid (Figure 3A). The concentrations of ethyl caprate catalyzed by An605 and An3131 were 52.36-fold and 40.26-fold higher than that of the negative control, respectively.

The optimal $\mathrm{pH}$ and $\mathrm{pH}$ stability were investigated. The optimum $\mathrm{pH}$ values of An605 and An3131 were both 4.0 (Figures 3B,C). Acidic conditions were suitable for esterification, while in $\mathrm{pH} 8.0$, the remaining enzymatic activities of An605 and An3131 were only $27.50 \pm 1.08 \%$ and $34.77 \pm 2.92 \%$, respectively, when compared with the enzyme activities in pH 4.0 (Figures 3D,E). Compared with An605, An3131 showed a poor performance about $\mathrm{pH}$ stability (Figure 3E). After incubating An605 and An3131 in a reaction buffer at $\mathrm{pH} 4.0$ for $1 \mathrm{~h}$, the residual enzyme activity of An605 was $91.60 \pm 4.44 \%$, and An3131 was $75.10 \pm 3.40 \%$, indicating An605 was much stable at $\mathrm{pH} 4.0$ compared with An3131 (Figures 3D,E). Temperature is also an important factor affecting enzyme activity. The optimal temperatures of An605 and An3131 were both $30^{\circ} \mathrm{C}$ (Figures 3F,G). When the temperature reached $70^{\circ} \mathrm{C}$, the relative enzyme activities of An605 and An3131 dropped to 57.75\% $\pm 2.66 \%$ and $62.27 \% \pm 4.98 \%$, respectively (Figures $3 F, G$ ). After incubating the pure enzyme solution at $30^{\circ} \mathrm{C}$ for $1 \mathrm{~h}$, the residual enzyme activity of An605 was $95.00 \pm 0.92 \%$ (Figure 3H), and that of An3131 was $87.80 \pm 4.92 \%$ (Figure 3I), indicating that both of the enzymes showed good performance toward temperature stability.

Metal ions and surfactants could affect enzyme activity. In this study, $\mathrm{Na}^{+}, \mathrm{Mg}^{2+}, \mathrm{Al}^{3+}, \mathrm{K}^{+}, \mathrm{Ca}^{2+}, \mathrm{Mn}^{2+}, \mathrm{Fe}^{2+}, \mathrm{Cu}^{2+}, \mathrm{Zn}^{2+}$, Tween 20, Trition X-100, and SDS with a final concentration of $2 \mathrm{mM}$ were added into the respective reaction mixtures to investigate their effects on enzyme activities. Results indicated that all the ions $\left(\mathrm{Al}^{3+}, \mathrm{Mg}^{2+}, \mathrm{Cu}^{2+}, \mathrm{K}^{+}, \mathrm{Na}^{+}, \mathrm{Zn}^{2+}, \mathrm{Mn}^{2+}\right.$, $\mathrm{Fe}^{2+}$, and $\mathrm{Ca}^{2+}$ ) had little effect on the enzyme activity of An605 (Figure 3J). The metal ion $\mathrm{Cu}^{2+}$ showed a slight inhibitory effect on the enzyme activity of An3131 (Figure 3K). Surfactants greatly inhibited the enzyme activities of An605 and An3131. After being added with Tween 20, Triton X-100, or SDS, the residual enzyme activities of An605 and An3131 were all lower than 20\% compared with the respective control (Figures 3J,K). Studies indicated that SDS could break the intramolecular and intermolecular hydrogen bonds of the enzyme, resulting in molecular unfolding, conformational change, and destruction of the enzyme (Zhang et al., 2018). Tween 20 and Triton X-100 are non-ionic surfactants, with a critical micelle concentration (CMC), that is, the concentration of aggregation to form micelles (Goswami, 2020). The CMC of Tween 20 was $0.06 \mathrm{mM}$, and the CMC of Triton X-100 was 0.2-0.9 mM (Goswami, 2020). The final concentration of the surfactant in the reaction system was $2.0 \mathrm{mM}$ and was higher than their respective CMCs. The formation of micelles would affect the interaction of the enzyme with substrates, thus generating inhibitory effects. 
TABLE 2 | Transcriptional level comparison of the genes from Aspergillus niger CGMCC 3.4309 under differentiated conditions.

\begin{tabular}{|c|c|c|c|c|}
\hline \multirow[t]{2}{*}{ Gene number (renumber) } & \multirow[t]{2}{*}{ Annotation } & \multicolumn{2}{|c|}{ Annotation pathway } & \multirow[t]{2}{*}{ Transcription level (Group 1 vs. Group 2)a } \\
\hline & & KEGG & GO & \\
\hline CL163.Contig2 (An163-2) & Lipase & K16815 & GO:0016042 & 3.21 \\
\hline CL163.Contig6 (An163-6) & Lipase & K16815 & GO:0016042 & 2.69 \\
\hline Unigene286 (An286) & Lipase & K14788 & GO:0016787 & 2.17 \\
\hline CL408 (An408) & Lipase & K16815 & GO:0016787 & 2.33 \\
\hline CL605 (An605) & Pectinesterase & K01051 & GO:0016829 & 4.36 \\
\hline CL923 (An923) & Carboxylesterase & NA & GO:0016787 & 2.39 \\
\hline CL1097 (An1097) & Esterase & K01050 & GO:0016787 & 3.74 \\
\hline Unigene1486 (An1486) & Lipase & K01050 & GO:0080030 & 2.32 \\
\hline CL1556.Contig4 (An1556-4) & Carboxylesterase & K02332 & GO:0016787 & 4.92 \\
\hline CL2017 (An2017) & Triglyceride lipase & K13333 & GO:0009395 & 3.76 \\
\hline CL2076 (An2076) & Acetylesterase/lipase & K01050 & GO:0016787 & 3.04 \\
\hline CL2505 (An2505) & Triglyceride lipase & K01956 & GO:0016787 & 2.43 \\
\hline Unigene2588 (An2588) & Esterase & K03927 & GO:0016787 & 2.06 \\
\hline CL2763 (An2763) & Esterase & K14675 & GO:0004806 & 2.32 \\
\hline Unigene2794 (An2794) & Lipase & K09252 & GO:0016787 & 2.85 \\
\hline CL3040.Contig1 (An3040-1) & Lipase & K16815 & GO:0004372 & 8.89 \\
\hline CL3040.Contig3 (An3040-3) & Lipase & K16815 & GO:0004372 & 7.69 \\
\hline CL3131 (An3131) & Esterase & K17648 & GO:0016787 & 2.27 \\
\hline CL3196 (An3196) & Lipase & K12389 & GO:0016787 & 2.36 \\
\hline Unigene3559 (An3559) & Lipase & K02332 & GO:0080030 & 2.40 \\
\hline Unigene3936 (An3936) & Carboxylesterase & K01049 & GO:0080030 & 3.40 \\
\hline Unigene5004 (An5004) & Lipase & NA & GO:0016042 & 2.95 \\
\hline Unigene5100 (An5100) & Feruloyl esterase & NA & GO:0016787 & 2.15 \\
\hline
\end{tabular}

${ }^{a}$ Group 1 is the species cultured under the optimized conditions, and Group 2 is the species cultured under the initial conditions.
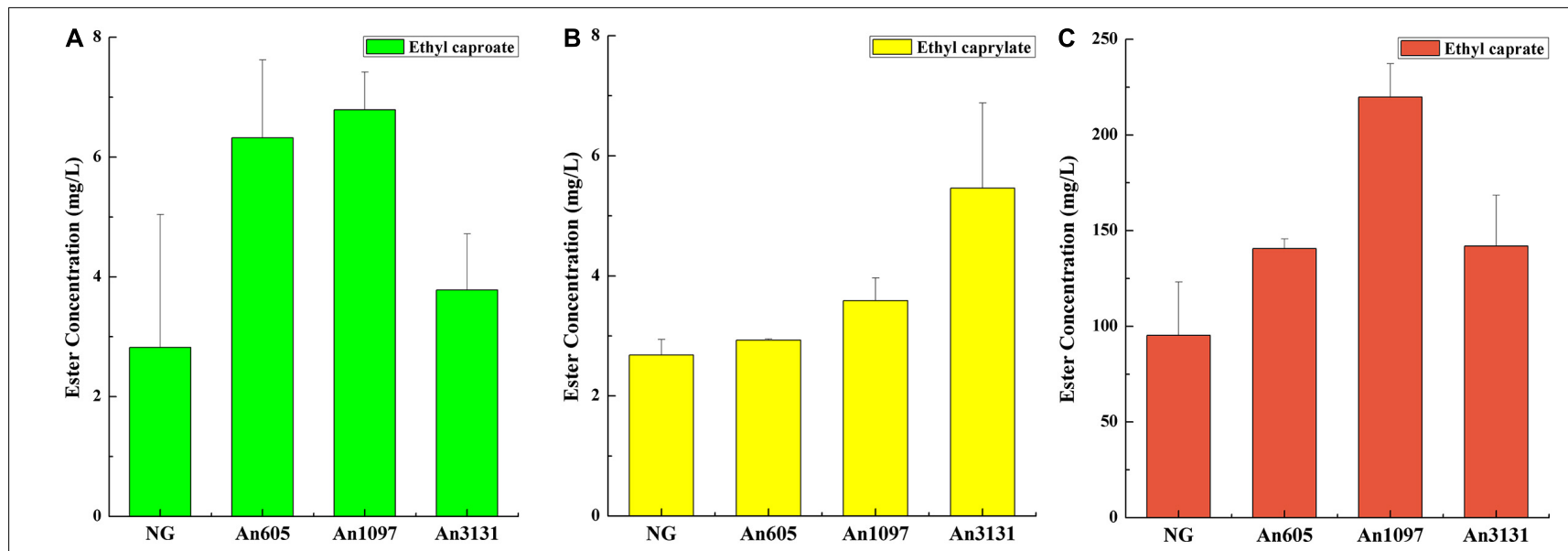

FIGURE 2 | Esterification property investigation of the enzymes from A. niger CGMCC 3.4309. (A) Synthesis of ethyl caproate. (B) Synthesis of ethyl caprylate. (C) Synthesis of ethyl caprate. NG, negative control, the crude enzyme solution of E. coli BL21/pET-28a(+); An605, the crude enzyme solution of E. coli BL21/pET-An605; An1097, the crude enzyme solution of E. coli BL21/pET-An1097; An3131, the crude enzyme solution of E. coli BL21/pET-An3131.

\section{Homology Modeling and Molecular Docking}

\section{Homology Modeling and Molecular Docking of An605, An1097, and An3131}

Homology modeling is a useful tool for investigating a protein structure. Combined with molecular docking, the possible enzyme-substrate interactions could be evaluated and provide useful information for the catalytic processes and for enabling interventions such as targeted mutagenesis (Tunyasuvunakool et al., 2021). Using An3131 as an example, the homology modeling was achieved using an alkaline esterase as the reference (PDB accession number 4YPV) (Pereira et al., 2017). The catalytic active sites were predicted by sequence alignment with 

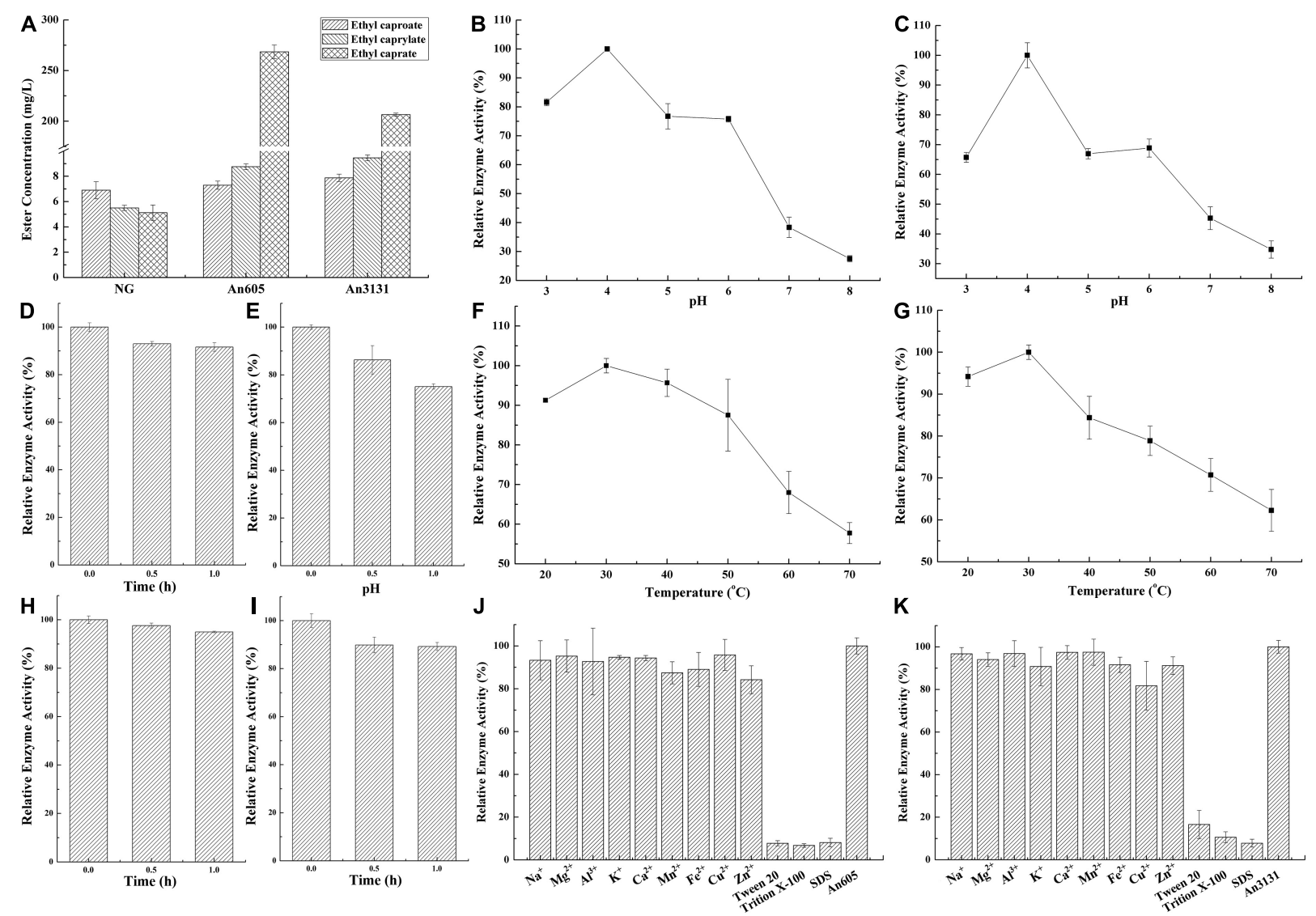

FIGURE 3 | Property analysis of the enzymes numbered An605 and An3131 from A. niger CGMCC 3.4309. (A) Substrate preference. NG, negative control, the reaction mixture without enzyme solution. (B) Optimal pH of An605. (C) Optimal pH of An3131. (D) pH stability of An605. (E) pH stability of An3131. (F) Optimal temperature of An605. (G) Optimal temperature of An3131. (H) Temperature stability of An605. (I) Temperature stability of An3131. (J) Effects of metal ions and common surfactants on enzyme activity of An605. (K) Effects of metal ions and common surfactants on enzyme activity of An3131.

the reference sequence of 4YPV (Supplementary Figure 4). An3131 contained a cap domain and the conserved sequence of $\mathrm{GX}_{1} \mathrm{SX}_{2} \mathrm{G}$ and was a candidate of the enzyme family of EC 3.1.1.1 (Figure 4A). The catalytic active center was constructed based on the spatial location of the catalytic active sites of the enzyme (Supplementary Figure 4). The molecular docking of An3131 with caproic acid, caprylic acid, capric acid, ethyl caproate, ethyl caprylate, and ethyl caprate were realized, respectively (Figure 4). Docking with capric acid indicated that the enzyme An3131 could interact with the ligand by hydrogen bonds through the residues Gly-124, Gly-125, Ser-196, and His-320 (Figure 4D). Ser-196 formed two hydrogen bonds with the two oxygen atoms $(\mathrm{C}=\underline{\mathrm{O}}$, and $\mathrm{C}-\underline{\mathrm{O}})$ of the carboxyl group of the substrate through the hydroxyl oxygen atom of Ser196 (Figure 4D). All the docking results were summarized inTable 3. The docking of An3131-capric acid showed the lowest binding energy, indicating the highest affinity of An3131 to capric acid, and was in accordance with the enzyme property analysis (Figure 3A). The other two enzymes were also docked with the fatty acids and ethyl fatty acids (Table 3). An1097 was also one of the enzymes that belonged to EC 3.1.1.1 through sequence alignment and molecular docking (Supplementary Figure 5 and
Table 3), while An605 was a candidate of the enzyme family EC 3.1.1.11 (pectinesterase) (Kent et al., 2016). The sequence alignment of An605 indicated that Gln-145, Gln-167, Asp-168, and Asp-189 might compose the catalytic center after comparing with the enzyme pectinesterase (PDB accession number $5 \mathrm{C} 1 \mathrm{E}$, identity 99.67\%) (Supplementary Figure 5a; Kent et al., 2016). No cap domain was reported of this enzyme that might affect catalysis. The results indicated that the catalytic processes for esterification were differentiated from the enzymes from $A$. niger CGMCC 3.4309.

\section{Homology Modeling and Molecular Docking of the Unexpressed Enzymes}

Due to the fact that only 11 of the 23 up-transcripted genes were heterologously expressed, the homology modeling and molecular docking of the left 12 enzymes not successfully expressed were carried out with the fatty acids and ethyl esters to further evaluate the esterification potential of these enzymes. Caproic acid, caprylic acid, capric acid, ethyl caproate, ethyl caprylate, and ethyl caprate were used as the ligands to dock with the enzymes, respectively. Six of the enzymes (An286, An1556-4, An2017, An2588, An3559, and An5004) built a 3D 

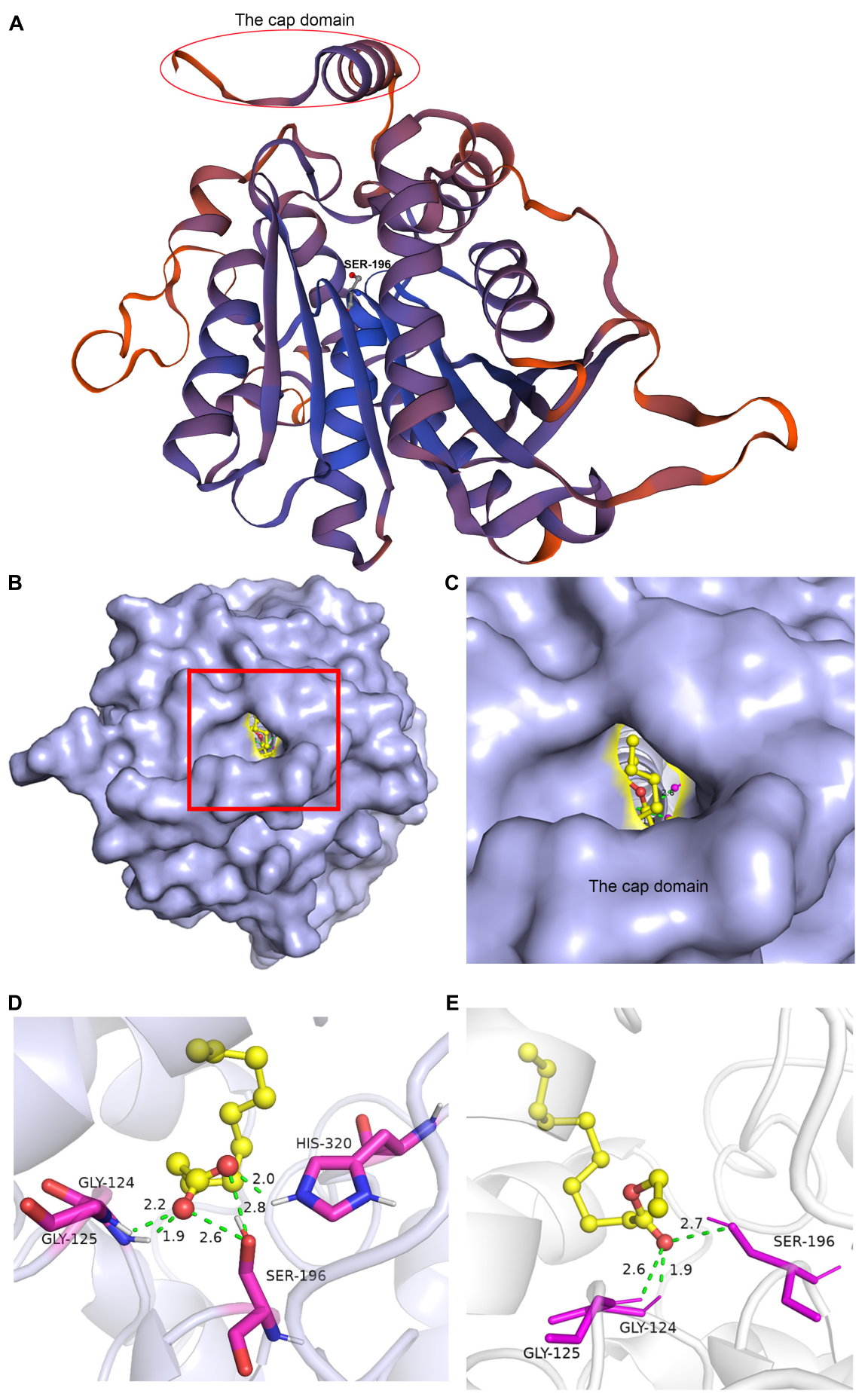

FIGURE 4 | Homology modeling of An3131 and molecular docking of An3131 with the ligands. (A) Homology modeling of An3131. (B) Molecular docking of An3131 with ethyl caprate. (C) The catalytic active center of An3131. (D) The interactions of the amino acids with capric acid. (E) The interactions of the amino acids with ethyl caprate.

structure after homology modeling using the proteins in the PDB database as references. All the ligands could dock to the catalytic regions of the respective enzymes and with varied interactions and binding energies (Supplementary Table 3). It is generally recognized that more interactions between the enzyme and the chemical molecules and the lower binding energies mean the higher affinity between enzyme and the chemical molecules (Williams et al., 2004; Liao et al., 2017). This increases the 


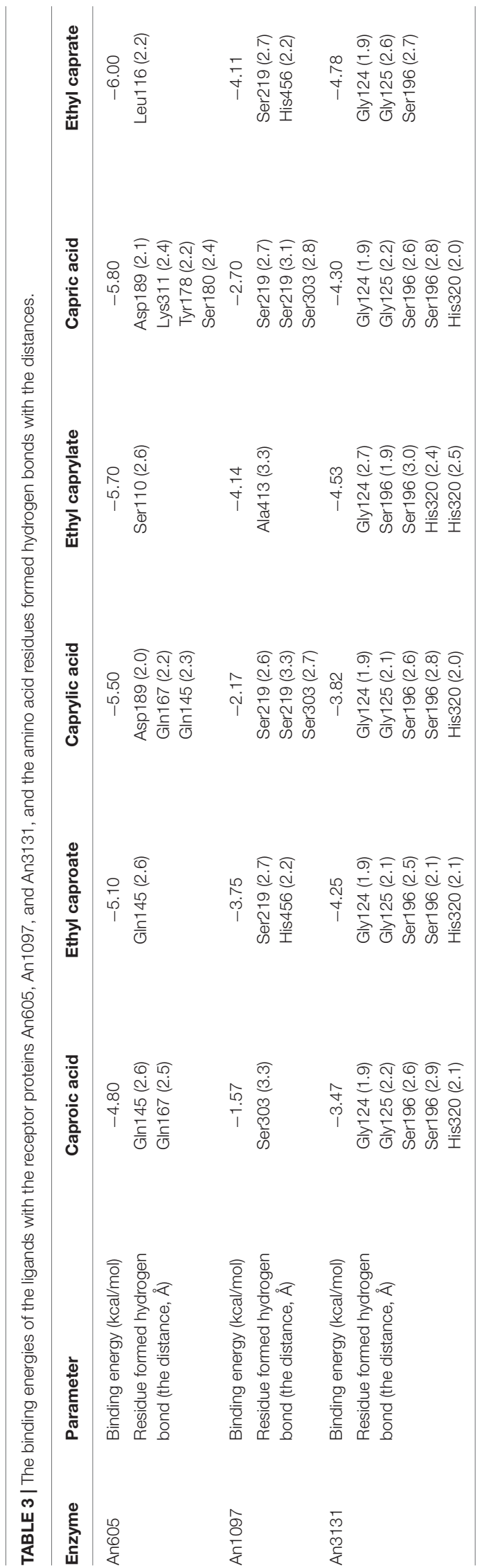

probability of enzymatic reaction. The formation of hydrogen bonds between the enzyme and the fatty acids or ethyl fatty acids, the distances, and binding energies were summarized in Supplementary Table 3.

The results indicated that An2017 showed a substrate preference toward caproic acid and caprylic acid; An1556-4 and An5004 showed a substrate preference toward caprylic acid; and An286, An2588, and An3559 showed a substrate preference toward capric acid according to the binding energy evaluation (Supplementary Table 3). Sequence alignment showed that all these six proteins harbored the conserves $\mathrm{GX}_{1} \mathrm{SX}_{2} \mathrm{G}$ sequence, and the catalytic active site was serine (Supplementary Figure 5). The left six enzymes An163-2, An163-6, An2505, An2763, An3040-1, and An3196 showed a quite-low sequence identity with the reference enzymes with known structures (less than $10 \%)$. RoseTTAFold was used for the structure prediction of these enzymes (Baek et al., 2021). As less information about the reference sequences, the catalytic active sites of these proteins needed to be further verified to predict their catalytic properties (Supplementary Figure 6).

The catalytic triad of enzymes that belonged to EC 3.1.1.1 or EC 3.1.1.3 was generally composed of Ser-His-Asp/Glu (Rauwerdink and Kazlauskas, 2015). The catalytic active region is usually covered by a cap domain (Yu et al., 2014; Rauwerdink and Kazlauskas, 2015). The alter of the cap domain exposed the catalytic active center and allowed the substrates to enter into the catalytic active center for esterification (Yu et al., 2014). About 3-5 $\AA$ around the hydroxyl group of serine was a groovelike structure, and a pair of hydrogen atoms from the amino acids could be used as hydrogen donors. The structure was usually called "oxygen anion hole," and was crucial to stabilize the transition complex formed during the catalytic process (Ferrer et al., 2015). The cavity between the oxygen anion hole and the key catalytic site serine was the substrate binding region.

According to the above analysis and literatures about enzymes that belonged to esterase/lipase, the enzymatic esterification reaction was a reversible process (Ferrer et al., 2015; Rauwerdink and Kazlauskas, 2015). When the cap domain altered, the substrate molecules entered into the catalytic active center of the enzyme (Figures 5A,B). The side chain hydroxyl oxygen atom of serine nucleophilically attacked the carboxyl carbon atom of the substrate. The electron was transferred to the carboxyl oxygen atom of the substrate to generate an oxygen anion and formed a hydrogen bond with the hydrogen atom of the oxygen anion hole to maintain the stability of enzyme-substrate complex. A covalent bond was formed between the carbon atom, substrate, and oxygen atom of the serine residue, generating a tetrahedral transition state 1 (Figures 5B,C). Thereafter, a water molecule was removed to form acyl-serine (Figures 5C,D). The alcohol entered into the catalytic active center and attacked the carbonyl carbon atom of acyl-serine to form tetrahedral transition state 2 (Figure 5E). Finally, the carbon atom of tetrahedral transition state 2 formed a covalent bond with the alcohol oxygen atom, and the covalent bond between the side chain hydroxyl oxygen atom of serine and the carbon atom of substrate was broken to realize the esterification reaction (Figure 5). Meanwhile, not all the esterification enzymes from $A$. niger CGMCC 3.4309 harbored 

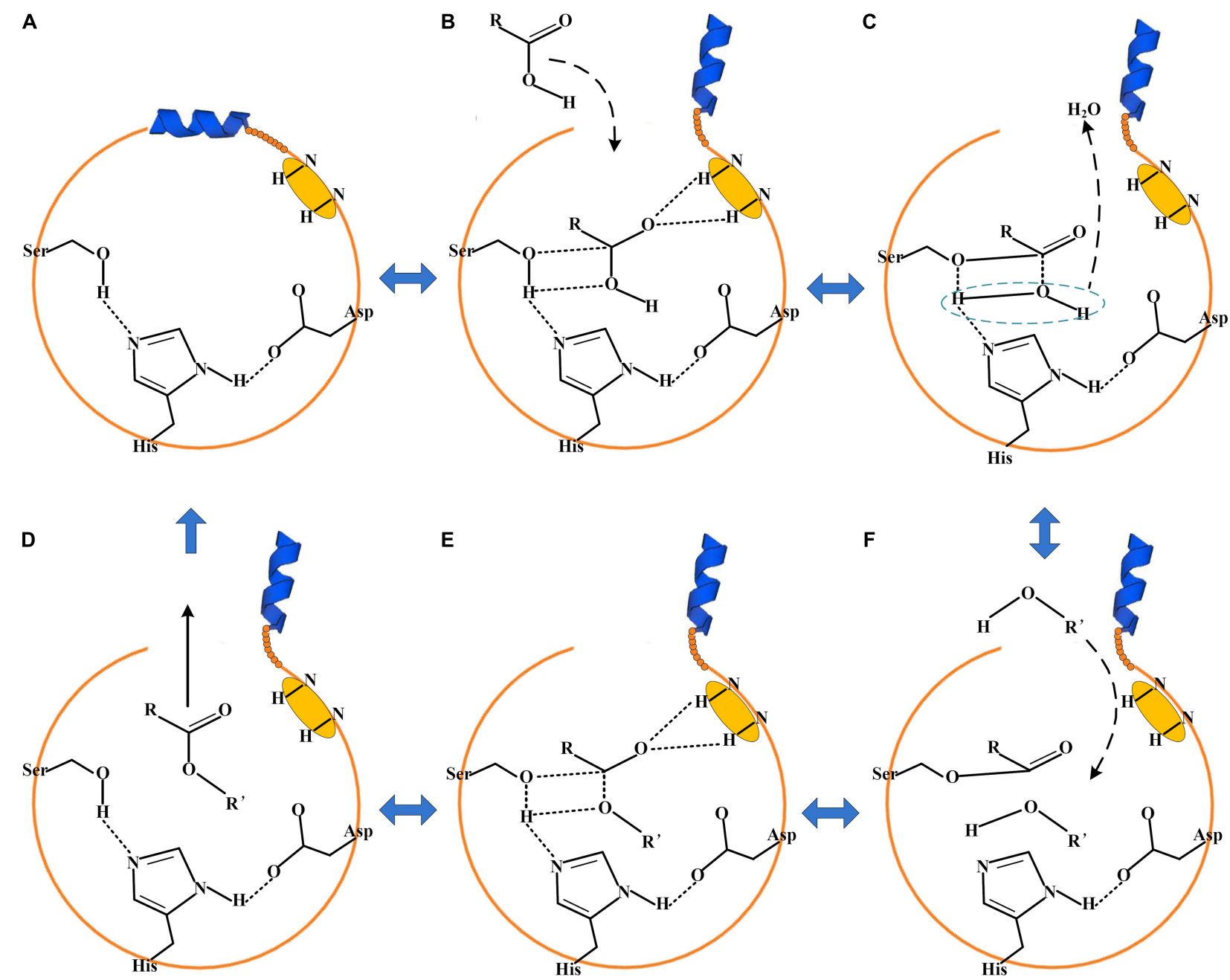

FIGURE 5 | The proposed mechanism for enzymatic esterification. (A) The cap domain of the enzyme was closed. (B) When the cap domain altered, the substrate acid entered into the catalytic active center. (C) Tetrahedral transition state 1. (D) The substrate alcohol entered into the catalytic active center. (E) Tetrahedral transition state 2. (F) Esterification completed.

the catalytic active residual serine like An605, indicating that the esterification mechanisms of the enzymes followed different catalytic mechanisms and needed to be further revealed.

Previous studies focused on ester synthesis using enzymes from $A$. niger, while most of the studies indicated that the enzymes preferred an organic-phase reaction system for esterification (Table 4). In an aqueous-phase reaction system, these enzymes mainly showed the performance on ester bond hydrolysis (Bourne et al., 2004). The sequences of the reported enzymes were differentiated with the enzymes in this work (Supplementary Figure 6), and the substrate preference was also distinctive. Based on differential culture conditions, transcriptome sequencing, gene library construction, enzymatic properties determination, and bioinformatics analysis, this work identified enzymes for fatty acid ethyl ester synthesis in aqueousphase systems for the first time. This provided a molecular basis for ester synthesis in an aqueous-phase system catalyzed by A. niger CGMCC 3.4309 .
In general, enzymatic ester synthesis in the organic-phase reaction system is more efficient than that in the aqueous-phase reaction system. However, for a fermented food manufacturing process, it is impossible to provide an organic-phase reaction condition. Therefore, it is of great significance to investigate the enzymatic synthesis of flavor esters under the aqueousphase reaction system. This can not only clarify the synthetic mechanism of flavor esters by $A$. niger but also helps to realize the stable synthesis of flavor substances through rational regulation of the fermentation process by microbes or enzymes, so as to ensure the product quality.

This work mainly focused on the catalytic synthesis of short- and medium-chain fatty acid ethyl esters according to the flavor ethyl esters in baijiu. This did not mean that the catalytic substrate spectrum of the strain and its related enzymes were limited to the substrates used in this work. Other substrates such as medium- and long-chain alcohols and fatty acids could be used as the substrates to further reveal 
TABLE 4 | Esterifying enzymes from Aspergillus niger and the characteristics.

\begin{tabular}{|c|c|c|c|c|}
\hline Enzyme name & Strain & $\begin{array}{l}\text { Accession } \\
\text { number }\end{array}$ & Characteristics & References \\
\hline Lipase & A. niger NCIM 1207 & - & $\begin{array}{l}\text { Esterification was carried out at } 30^{\circ} \mathrm{C} \text { in } 25 \mathrm{~mL} \text { stoppered flasks } \\
\text { with } 10 \mathrm{ml} n \text {-hexane containing fusel oil }(1.9 \mathrm{~mL}) \text {, acetic acid } \\
\text { ( } 360 \mathrm{uL} \text { ) and } 1 \mathrm{~g} \text { enzyme. the product concentration of isoamyl } \\
\text { acetate was } 50.0 \pm 2.8 \mathrm{~g} / \mathrm{L} \text { in } 96 \mathrm{~h} \text {. }\end{array}$ & Mhetras et al., 2010 \\
\hline Lipase & A. niger F0215 & An14g02170 & $\begin{array}{l}\text { The ethyl lactate, butyl butyrate, and ethyl caprylate flavor } \\
\text { esters were produced by esterification of the corresponding } \\
\text { acids with conversion efficiencies of } 15.8 \%, 37.5 \% \text {, and } 24.7 \% \text {, } \\
\text { respectively, in a soybean oil-based solvent system. }\end{array}$ & Cong et al., 2019 \\
\hline Ferulic acid esterase & A. niger & - & $\begin{array}{l}\text { The esterification reaction mixture contained } 1 \% \text { ferulic acid in } \\
\text { the presence of } 85 \% \text { glycerol and } 5 \% \text { dimethyl sulfoxide at } \mathrm{pH} \\
4.0 \text { acetate buffer and } 50^{\circ} \mathrm{C} \text {, and } 81 \% \text { of ferulic acid could be } \\
\text { converted to } 1 \text {-glyceryl ferulate. }\end{array}$ & Tsuchiyama et al., 2006 \\
\hline EstA & A. niger & AY456379 & $\begin{array}{l}\text { EstA could hydrolyze vinyl acetate with } k_{\text {cat }} \text { and } K_{m} \text { of } 400 \text { and } \\
2.7 \text {, respectively; vinyl propionate with } k_{\text {cat }} \text { and } K_{m} \text { of } 160 \text { and } \\
2.9 \text {, respectively; triacetin with } k_{\text {cat }} \text { and } K_{m} \text { of } 265 \text { and } 15 \text {, } \\
\text { respectively, at } 25^{\circ} \mathrm{C}, \mathrm{pH} 5.0 \text { and } 0.1 \mathrm{M} \mathrm{NaCl} \text { buffer. }\end{array}$ & Bourne et al., 2004 \\
\hline Lipase & A. niger & - & $\begin{array}{l}\text { AN0512Lip exhibited high tolerance for various polar organic } \\
\text { solvents with logP }<0.8 \text {, and the highest lipase activity }(476 \% \\
\text { of its original activity) was achieved after addition of } 90 \%(\mathrm{v} / \mathrm{v}) \\
\text { isopropanol to the reaction mixture. AN0512Lip also displayed } \\
\text { 3-regiospecificity and great affinity for the long-chain fatty ester. }\end{array}$ & Liu et al., 2015 \\
\hline Feruloyl esterase & A. niger & - & $\begin{array}{l}\text { Suitable conditions for esterification of ferulic acid with } \\
\text { diglycerol were } 100 \mathrm{mg} \text { of ferulic acid in the presence of } 1 \mathrm{~g} \text { of } \\
\text { diglycerol and } 0.1 \mathrm{~mL} \text { of } 1 \mathrm{M} \text { phosphate buffer (pH 6.0) at } 50^{\circ} \mathrm{C} \\
\text { under reduced pressure. Under these conditions, } 168 \mathrm{mg} \text { of } \\
\text { feruloyl diglycerols was obtained, corresponding to a } 95 \% \\
\text { conversion rate of ferulic acid. }\end{array}$ & Kikugawa et al., 2012 \\
\hline
\end{tabular}

the esterification ability of the strain and related enzymes. These investigations will promote the application of $A$. niger CGMCC 3.4309 and its enzymes for flavor ester synthesis in food industry.

\section{CONCLUSION}

A strain of $A$. niger-designated CGMCC 3.4309 was characterized to efficiently synthesize fatty acid ethyl esters in an aqueous-phase reaction system. The optimal culture conditions for producing esterifying enzymes included a culture temperature of $28^{\circ} \mathrm{C}$ and rotation rate of $250 \mathrm{r} / \mathrm{min}$, with the culture medium composed of lactose as the carbon source and $\left(\mathrm{NH}_{4}\right)_{2} \mathrm{SO}_{4}$ as the nitrogen source. The conversion ratios of ethyl valerate, ethyl caproate, ethyl caprylate, and ethyl caprate reached 7.87, 29.2, 94.8 , and $85.2 \%$, respectively. Compared with the initial cultural conditions, the conversion ratios of the above four esters were increased by $3.53,5.97,2.14$, and 3.02 folds, respectively.

Based on transcriptome sequencing, gene library construction, enzymatic property analysis, homology modeling, and molecular docking, three enzymes with ester synthetic abilities were identified, and the ester synthetic potentials of other enzymes were evaluated. For example, the enzyme numbered An2017, An1556-4, and An5004 might have the ability to catalyze the synthesis of ethyl caprylate. The possible catalytic mechanism of esterification was proposed. These studies provided a technological combination for a comprehensive analysis of the ester synthetic enzymes in aqueous phase derived from microorganisms during the baijiu manufacturing process.

\section{NCBI NUCLEOTIDE ACCESSION}

The RNA sequencing data were submitted to NCBI Sequence Read Archive under accession numbers (SRX12524232), (SRR16244411), (SRX12524233), (SRR16244410), (SRX12524234), and (SRR16244409) for Group 1 samples and (SRX12524688), (SRR16244867), (SRX12524689), (SRR16244866), (SRX12524690), and (SRR16244865) for Group 2 samples.

\section{DATA AVAILABILITY STATEMENT}

The datasets presented in this study can be found in online repositories. The names of the repository/repositories and accession number(s) can be found in the article/Supplementary Material.

\section{AUTHOR CONTRIBUTIONS}

YX contributed to the methodology, investigation, data curation, writing-original draft, and funding acquisition. $\mathrm{HH}$ and MW contributed to the methodology and investigation. HL contributed to the data curation and writing-original draft. ML, 
CSZ, ZZ, CNZ, and WL contributed to the data curation. XL and BS contributed to the resources, writing-review and editing, funding acquisition, and supervision. All authors contributed to the article and approved the submitted version.

\section{FUNDING}

This work was supported by National Natural Science Foundation of China (Grant Numbers 31830069, 31801467, and 32072165), Beijing Municipal Natural Science Foundation and Beijing Municipal Education Commission (Grant Numbers KM202110011003 and KZ202010011018), School

\section{REFERENCES}

Audic, S., and Claverie, J. M. (1997). The significance of digital gene expression profiles. Genome Res. 7, 986-995. doi: 10.1101/gr.7.10.986

Baek, M., DiMaio, F., Anishchenko, I., Dauparas, J., Ovchinnikov, S., Lee, G. R., et al. (2021). Accurate prediction of protein structures and interactions using a three-track neural network. Science 373, 871-876. doi: 10.1126/science.abj8754

Bollinger, A., Molitor, R., Thies, S., Koch, R., Coscolin, C., Ferrer, M., et al. (2020). Organic-solvent-tolerant carboxylic ester hydrolases for organic synthesis. Appl. Environ. Microbiol. 86, e106-e120. doi: 10.1128/AEM.00106-20

Bourne, Y., Hasper, A. A., Chahinian, H., Juin, M., de Graaff, L. H., and Marchot, P. (2004). Aspergillus niger protein EstA defines a new class of fungal esterases within the alpha/beta hydrolase fold superfamily of proteins. Structure 12, 677-687. doi: 10.1016/j.str.2004.03.005

Chung, H., Lee, N., Seo, J. A., and Kim, Y. S. (2017). Comparative analysis of nonvolatile and volatile metabolites in Lichtheimia ramosa cultivated in different growth media. Biosci. Biotechnol. Biochem. 81, 565-572. doi: 10.1080/ 09168451.2016 .1256756

Cong, S. Z., Tian, K. M., Zhang, X., Lu, F. P., Singh, S., Prior, B., et al. (2019). Synthesis of flavor esters by a novel lipase from Aspergillus niger in a soybeansolvent system. Biotech 9:244. doi: 10.1007/s13205-019-1778-5

Fan, G. S., Liu, P. X., Chang, X., Yin, H., Cheng, L. J., Teng, C., et al. (2021). Isolation and identification of a high-yield ethyl caproate-producing yeast from daqu and optimization of its fermentation. Front. Microbiol. 12:663744. doi: $10.3389 /$ fmicb.2021.663744

Fan, G. S., Zhu, Y. T., Fu, Z. L., Sun, B. G., Teng, C., Yang, R., et al. (2020). Optimization of fermentation conditions for the production of recombinant feruloyl esterase from Burkholderia pyrrocinia B1213. Biotech 10:216. doi: 10. 1007/s13205-020-02198-1

Feng, Y., He, Z., Ong, S. L., Hu, J., Zhang, Z., and Ng, W. J. (2003). Optimization of agitation, aeration, and temperature conditions for maximum $\beta$-mannanase production. Enzy. Microbial. Technol. 32, 282-289. doi: 10.1016/ S0141-0229(02)00287-9

Ferrer, M., Bargiela, R., Martinez-Martinez, M., Mir, J., Koch, R., Golyshina, O. V., et al. (2015). Biodiversity for biocatalysis: A review of the alpha/betahydrolase fold superfamily of esterases-lipases discovered in metagenomes. Biocatal. Biotransform. 33, 235-249. doi: 10.3109/10242422.2016.1151416

Goswami, D. (2020). Lipase catalysis in presence of nonionic surfactants. Appl. Biochem. Biotechnol. 191, 744-762. doi: 10.1007/s12010-019-03212-w.

Holm, L., and Laakso, L. M. (2016). Dali server update. Nucleic Acids Res. 44, W351-W355. doi: 10.1093/nar/gkw357

Hong, J. X., Zhao, D. R., and Sun, B. G. (2021). Research progress on the profile of trace components in Baijiu. Food Rev. Int. 137:109695. doi: 10.1080/87559129. 2021.1936001

Jain, D., and Katyal, P. (2018). Optimization of gluco-amylase production from Aspergillus spp. for its use in saccharification of liquefied corn starch. Biotech 8:101. doi: $10.1007 / \mathrm{s} 13205-018-1131-4$

Jin, G. Y., Zhu, Y., and Xu, Y. (2017). Mystery behind Chinese liquor fermentation. Trends Food Sci. Technol. 63, 18-28. doi: 10.1016/j.tifs.2017.02.016
Level Cultivation Foundation of Beijing Technology and Business University for Distinguished and Excellent Young Scholars (Grant Number BTBUYP2021), and Key Laboratory of Wuliangye-flavor Liquor Solid-state Fermentation, China National Light Industry (Grant Number 2021JJ006).

\section{SUPPLEMENTARY MATERIAL}

The Supplementary Material for this article can be found online at: https://www.frontiersin.org/articles/10.3389/fmicb. 2021.820380/full\#supplementary-material

Kent, L. M., Loo, T. S., Melton, L. D., Mercadante, D., Williams, M. A. K., and Jameson, G. B. (2016). Structure and properties of a non-processive, saltrequiring, and acidophilic pectin methylesterase from Aspergillus niger provide insights into the key determinants of processivity control. J. Biol. Chem. 291, 1289-1306. doi: 10.1074/jbc.M115.673152

Kikugawa, M., Tsuchiyama, M., Kai, K., and Sakamoto, T. (2012). Synthesis of highly water-soluble feruloyl diglycerols by esterification of an Aspergillus niger feruloyl esterase. Appl. Microbiol. Biotechnol. 95, 615-622. doi: 10.1007/s00253012-4056-6

Kruis, A. J., Bohnenkamp, A. C., Patinios, C., van Nuland, Y. M., Levisson, M., Mars, A. E., et al. (2019). Microbial production of short and medium chain esters: Enzymes, pathways, and applications. Biotechnol. Adv. 37:107407. doi: 10.1016/j.biotechadv.2019.06.006

Kruis, A. J., Levisson, M., Mars, A. E., van der Ploeg, M., Daza, F. G., Ellena, V., et al. (2017). Ethyl acetate production by the elusive alcohol acetyltransferase from yeast. Metabol. Eng. 41, 92-101. doi: 10.1016/j.ymben.2017. 03.004

Li, B., and Dewey, C. N. (2011). RSEM: accurate transcript quantification from RNA-Seq data with or without a reference genome. BMC Bioinform. 12:323. doi: 10.1186/1471-2105-12-323

Liao, K. B., Pickel, T. C., Oyarskikh, V. B., Acsa, J. B., Usaev, D. G. M., and Davies, H. M. L. (2017). Site-selective and stereoselective functionalization of non-activated tertiary C-H bonds. Nature 551, 609-613. doi: 10.1038/nature 24641

Liu, G., Hu, S. Q., Li, L., and Hou, Y. (2015). Purification and Characterization of a lipase with high thermostability and polar organic solvent-tolerance from Aspergillus niger AN0512. Lipids 50, 1155-1163. doi: 10.1007/s11745-0154052-6

Mhetras, N., Patil, S., and Gokhale, D. (2010). Lipase of Aspergillus niger NCIM 1207: A potential biocatalyst for synthesis of isoamyl acetate. Indian J. Microbiol. 50, 432-437. doi: 10.1007/s12088-011-0087-4

Pereira, M. R., Maester, T. C., Mercaldi, G. F., de Macedo Lemos, E. G., Hyvonen, M., and Balan, A. (2017). From a metagenomic source to a high-resolution structure of a novel alkaline esterase. Appl. Microbiol. Biotechnol. 101, 49354949. doi: 10.1007/s00253-017-8226-4

Pertea, G., Huang, X. Q., Liang, F., Antonescu, V., Sultana, R., Karamycheva, S., et al. (2003). TIGR Gene Indices clustering tools (TGICL): A software system for fast clustering of large EST datasets. Bioinformatics 19, 651-652. doi: 10.1093/bioinformatics/btg034

Rauwerdink, A., and Kazlauskas, R. J. (2015). How the same core catalytic machinery catalyzes 17 different reactions: the serine-histidine-aspartate catalytic triad of alpha/beta-hydrolase fold enzymes. ACS Cataly. 5, 6153-6176. doi: 10.1021/acscatal.5b01539

Sun, J. C., and Liu, S. Q. (2015). Ester synthesis in aqueous media by lipase: Alcoholysis, esterification and substrate hydrophobicity. J. Food Biochem. 39, 11-18. doi: 10.1111/jfbc.12104

Sun, J. C., Yu, B., Curran, P., and Liu, S. Q. (2012). Optimisation of flavour ester biosynthesis in an aqueous system of coconut cream and fusel oil catalysed by lipase. Food Chem. 135, 2714-2720. doi: 10.1016/j.foodchem.2012.06.119 
Tang, M. C., He, H. Y., Zhang, F., and Tang, G. L. (2013). Baeyer-Villiger oxidation of acyl carrier protein-tethered thioester to acyl carrier proteinlinked thiocarbonate catalyzed by a monooxygenase domain in FR901464 biosynthesis. ACS Cataly. 3, 444-447. doi: 10.1021/cs300819e

Tsuchiyama, M., Sakamoto, T., Fujita, T., Murata, S., and Kawasaki, H. (2006). Esterification of ferulic acid with polyols using a ferulic acid esterase from Aspergillus niger. Biochimica Et Biophysica Acta-Gen. Sub. 1760, 1071-1079. doi: 10.1016/j.bbagen.2006.03.022

Tunyasuvunakool, K., Adler, J., Wu, Z., Green, T., Zielinski, M., Zidek, A., et al. (2021). Highly accurate protein structure prediction for the human proteome. Nature 596, 590-596. doi: 10.1038/s41586-021-03828-1

Wang, B. W., Wu, Q., Xu, Y., and Sun, B. G. (2020). Synergistic effect of multiple saccharifying enzymes on alcoholic fermentation for Chinese Baijiu production. Appl. Environ. Microbiol. 86, e13-e20. doi: 10.1128/AEM.00013-20

Wang, B. W., Wu, Q., Xu, Y., and Sun, B. G. (2021). Multiple sugars promote microbial interactions in Chinese baijiu fermentation. LWT-Food Sci. Technol. 138:110631. doi: 10.1016/j.lwt.2020.110631

Wei, Y., Zou, W., Shen, C. H., and Yang, J. G. (2020). Basic flavor types and component characteristics of Chinese traditional liquors: A review. J. Food Sci. 85, 4096-4107. doi: 10.1111/1750-3841.15536

Williams, D. H., Stephens, E., O’Brien, D. P., and Zhou, M. (2004). Understanding noncovalent interactions: Ligand binding energy and catalytic efficiency from ligand-induced reductions in motion within receptors and enzymes. Angewandte Chemie-Int. Ed. 43, 6596-6616. doi: 10.1002/anie.200300644

Wu, J. H., Liu, Y., Zhao, H., Huang, M. Q., Sun, Y., Zhang, J. L., et al. (2021). Recent advances in the understanding of off-flavors in alcoholic beverages: Generation, regulation, and challenges. J. Food Compos. Anal. 103:104117. doi: 10.1016/j.jfca.2021.104117

Wu, Q., Chen, B., and Xu, Y. (2015). Regulating yeast flavor metabolism by controlling saccharification reaction rate in simultaneous saccharification and fermentation of Chinese Maotai-flavor liquor. Int. J. Food Microbiol. 200, 39-46. doi: 10.1016/j.ijfoodmicro.2015.01.012

Xu, C., Sun, B., Xu, Y., Fan, G., and Li, X. (2020). Identification of ethyl hexanoate synthesis esterifying enzymes production strain and its enzyme production conditions optimization. J. Chinese Inst. Food Sci. Technol. 20, 138-147. doi: 10.16429/j.1009-7848.2020.05.018

Xu, Y. Q., Sun, B. G., Fan, G. S., Teng, C., Xiong, K., Zhu, Y. P., et al. (2017). The brewing process and microbial diversity of strong flavour Chinese spirits: A review. J. Inst. Brew. 123, 5-12. doi: 10.1002/jib.404

Xu, Y. Q., Liu, X., Zhao, J. R., Huang, H. Q., Wu, M. Q., Li, X. T., et al. (2021a). An efficient phthalate ester-degrading Bacillus subtilis: Degradation kinetics, metabolic pathway, and catalytic mechanism of the key enzyme. Environ. Pollut. 273:116461. doi: 10.1016/j.envpol.2021.116461

Xu, Y. Q., Wang, X. C., Liu, X., Li, X. T., Zhang, C. N., Li, W. W., et al. (2021b). Discovery and development of a novel short-chain fatty acid ester synthetic biocatalyst under aqueous phase from Monascus purpureus isolated from Baijiu. Food Chem. 338:128025. doi: 10.1016/j.foodchem.2020.128025
Xu, Y. Q., Zhao, J. R., Huang, H. Q., Guo, X. Y., Li, X. T., Zou, W., et al. (2022a). Biodegradation of phthalate esters by Pantoea dispersa BJQ0007 isolated from Baijiu. J. Food Comp. Anal. 105:104201. doi: 10.1016/j.jfca.2021.104201

Xu, Y. Q., Zhao, J. R., Liu, X., Zhang, C. S., Zhao, Z. G., Li, X. T., et al. (2022b). Flavor mystery of Chinese traditional fermented baijiu: The great contribution of ester compounds. Food Chem. 369:130920. doi: 10.1016/j.foodchem.2021. 130920

Xu, Y. Q., Zhu, Y., Li, X. T., and Sun, B. G. (2020). Dynamic balancing of intestinal short-chain fatty acids: The crucial role of bacterial metabolism. Trends Food Sci. Technol. 100, 118-130. doi: 10.1016/j.tifs.2020. 02.026

Ye, H., Wang, J., Shi, J., Du, J. Y., Zhou, Y. H., Huang, M. Q., et al. (2021). Automatic and intelligent technologies of solid-state fermentation process of baijiu production: Applications, challenges, and prospects. Foods 10:680. doi: 10.3390/foods 10030680

Yu, X. W., Zhu, S. S., Xiao, R., and Xu, Y. (2014). Conversion of a Rhizopus chinensis lipase into an esterase by lid swapping. J. Lipid Res. 55, 1044-1051. doi: 10.1194/jlr.M043950

Yurimoto, H., Kato, N., and Sakai, Y. (2005). Assimilation, dissimilation, and detoxification of formaldehyde, a central metabolic intermediate of methylotrophic metabolism. Chem. Rec. 5, 367-375. doi: 10.1002/tcr. 20056

Zhang, R., Liu, Y., Huang, X. R., Xu, M. C., Liu, R. T., and Zong, W. S. (2018). Interaction of a digestive protease, Candida rugosa lipase, with three surfactants investigated by spectroscopy, molecular docking and enzyme activity assay. Sci. Total Environ. 622, 306-315. doi: 10.1016/j.scitotenv.2017.11.305

Zou, W., Ye, G. B., and Zhang, K. Z. (2018). Diversity, function, and application of clostridium in chinese strong flavor baijiu ecosystem: A review. J. Food Sci. 83, 1193-1199. doi: 10.1111/1750-3841.14134

Conflict of Interest: The authors declare that the research was conducted in the absence of any commercial or financial relationships that could be construed as a potential conflict of interest.

Publisher's Note: All claims expressed in this article are solely those of the authors and do not necessarily represent those of their affiliated organizations, or those of the publisher, the editors and the reviewers. Any product that may be evaluated in this article, or claim that may be made by its manufacturer, is not guaranteed or endorsed by the publisher.

Copyright (C) $2022 \mathrm{Xu}$, Huang, Lu, Wu, Lin, Zhang, Zhao, Li, Zhang, Li and Sun. This is an open-access article distributed under the terms of the Creative Commons Attribution License (CC BY). The use, distribution or reproduction in other forums is permitted, provided the original author(s) and the copyright owner(s) are credited and that the original publication in this journal is cited, in accordance with accepted academic practice. No use, distribution or reproduction is permitted which does not comply with these terms. 